\title{
Relative Therapeutic Efficacy of Some Vertebral Mobilization Techniques in the Management of Unilateral Cervical Spondylosis: A Comparative Study
}

J. Phys. Ther. Sci.

20: 103-108, 2008

\author{
Michael OgBonnia EgWU, BMR, MSc, PhD ${ }^{1)}$ \\ 1) Department of Medical Rehabilitation, Faculty of Basic Medical Sciences, College of Health \\ Sciences, Obafemi Awolowo University: Ile-Ife Nigeria. \\ TEL +234 8033739499, E-mail: egwumo@yahoo.com
}

\begin{abstract}
Purpose] This study compared the relative efficacy of Posterior-Anterior Unilateral Pressure (PAUP), Anterior-Posterior Unilateral Pressure (APUP), Cervical Oscillatory Rotation (COR) and Transverse Oscillatory Pressure (TOP) techniques of manual therapy in the management of unilateral cervical spondylosis. [Subjects and Methods] Twenty-four male subjects in each of four groups (96 patients; age range 40-50 years, mean $44 \pm 5.3$ years) received treatment 3 times per week for four weeks. Mean treatment time, pain recovery pattern (pain-free, residual pain, fair improvement, no change or worse) and relapse after 3 months were outcome measures. [Results] Significantly $(\mathrm{P}<0.001)$ less time was spent applying APUP (130 min) and PAUP (156 min) than COR (192 min) and TOP (219 min) with significantly $(\mathrm{P}<0.001)$ more pain-free patients in APUP $(63 \%)$ and PAUP $(46 \%)$ groups than in COR (17\%) and TOP (25\%) groups. Relapse rates after 3 months were $8 \%$ (TOP) and $12 \%$ (COR) and these treatments had no effect on symptoms of $4 \%$ and $8 \%$ of patients, respectively. [Conclusion] APUP and PAUP achieve faster pain relief in more patients with unilateral cervical spondylosis than rotation and transverse pressure. Unilateral pressures should be preferred techniques of manual therapy for patients with unilateral neck pain and APUP promise a better outcome.
\end{abstract}

Key words: Unilateral cervical spondylosis, Manual therapy techniques, Relative efficacy

(This article was submitted Oct. 3, 2007, and was accepted Nov. 30, 2007)

\section{INTRODUCTION}

Cervical Spondylosis (CS) connotes progressive degeneration of the intervertebral discs leading to changes in the surrounding structures especially the bones and meninges ${ }^{1)}$. Its signs and symptoms fall into two main groups: Those due to root compression or cervical radiculopathy and those due to cord compression or cervical myelopathy'). In addition, other related symptoms such as parasthesia, limitation of neck movements, headache, pain in the neck and the symptoms of vertebro-basilar insufficiency may be present ${ }^{1,3)}$. These signs and symptoms may occur singly or in any combination and may affect the vertebral bodies, intervertebral discs, facet joints, longitudinal ligaments and ligamentum flavum ${ }^{4}$. However, it has been observed that in adults older than 40 years, about $60 \%$ have degenerative disc disease, while $20 \%$ have foraminal stenosis both of which may irritate nociceptors ${ }^{1,5)}$. Furthermore, advanced spondylotic changes can narrow the vertebebral and intervertebral foramina and restrict cervical mobility resulting in pain and dysfunction ${ }^{4)}$. 
The management of CS includes the use of cervical collars, traction, heat, active neck exercises, massage and neck posture instructions ${ }^{1,6,7)}$. Manual therapy is also one of the modalities used in combating this condition when it is of clear mechanical origin ${ }^{4-7)}$. Whereas various techniques of manual therapy are routinely used to treat neck disorders, their therapeutic efficacies remain largely empirical $^{5,8,9)}$. Snodgrass et al. ${ }^{5)}$ studied manual forces applied by therapists during posterioranterior mobilization of the cervical spine and found that manual forces applied during this therapy vary widely among therapists. This finding suggests that using the same technique, different therapists may obtain variable results due to varying degrees of proficiency in the technique.

Apart from the skill of the therapist, the choice of technique may also affect the therapeutic outcome of manual therapy ${ }^{4,5,8)}$. Over the years several techniques of manual therapy have been proposed for the treatment of CS (cervical spondylosis), some of which include translatoric mobilization ${ }^{4)}$, Sustained Natural Apophyseal Glides ("SNAGS") techniques ${ }^{10)}$, vertical oscillatory pressure ${ }^{6,11)}$, Transverse Oscillatory Pressure (TOP), Cervical Oscillatory Rotation (TOP), Posterior-Anterior Unilateral Pressure (PAUP) and Anterior-Posterior Unilateral Pressure (APUP) ${ }^{6,7)}$. Some of these techniques are perceived to be simpler and/or more effective than the others for unilateral and bilateral symptoms, respectively, however comparative studies evaluating their relative efficacy are scant. The purpose of this study was to examine the relative efficacy of four techniques of vertebral mobilization, PAUP, APUP, COR, and TOP in the management of unilateral CS. The working hypothesis of this study was that there would be no significant difference in Treatment Time (TT), pain recovery and relapse pattern in patients with unilateral CS receiving manual therapy.

\section{METHODS AND PROCEDURE}

Ninety-six male patients (age range $40-50$ years, mean $44 \pm 5.3$ years) with diagnosis of CS referred for manipulative therapy to E.M.O Physiotherapy Clinic and the Manual Therapy Laboratory, Department of Medical Rehabilitation, Obafemi Awolowo University, both in Ile-Ife, Nigeria, were the study sample. The total number represents the male patients referred for treatment who gave their consent to participate in the study over a five year period (2002-2006) and met the inclusion criteria described below:

i. All had severe neck pain as subjectively defined by the patients;

ii. Pain was unilaterally distributed relative to the midline of the neck with brachial symptoms of $\mathrm{C} 5 / 6$ distribution;

iii. Skin rolling test was positive ${ }^{6}$, and digital pressure over these levels was also sore, suggestive of a positive test ${ }^{7,12)}$;

iv. None of the patients had received manual therapy for neck pain;

v. The onset of pain in all the cases was within six weeks of entry into this study;

vi. There was no history of vertebro-basilar insufficiency in any of the patients ${ }^{3)}$;

vii. Age of the patients was between 40 and 50 years.

The patients were selected prospectively and only those who demonstrated the above characteristics were included in the study. Patients were slotted sequentially into four groups. The first patient was slotted into group one and was treated using posterior-anterior-unilateral pressure; the second patient was slotted into group two and was treated using antero - posterior unilateral pressure; the third patient went into the third group which received cervical oscillatory rotation; and the fourth patient was slotted into the fourth group which was treated using transverse oscillatory pressure, while the fifth patient was again slotted into group one and was treated accordingly. This pattern was maintained until the data of 24 subjects was obtained for each group. The exact treatment technique to be used was explained to the patient and those that gave their consent after explanations were recruited.

The methods of application of the various techniques in the appropriate groups are described below:

Group 1 (Fig. 1): The Posterior-AnteriorUnilateral Pressure (PAUP)

PAUP was administered with the patient lying prone, his forehead on his superimposed hands as described by Maitland ${ }^{7)}$. Oscillatory pressure was directed through the thumbs of the therapist postero-anteriorly immediately over the transverse process on the effected side. After eliciting pain through thumb pressure (digital pressure), treatment consisted of nudging one grade ${ }^{6,7)}$ into, but not 


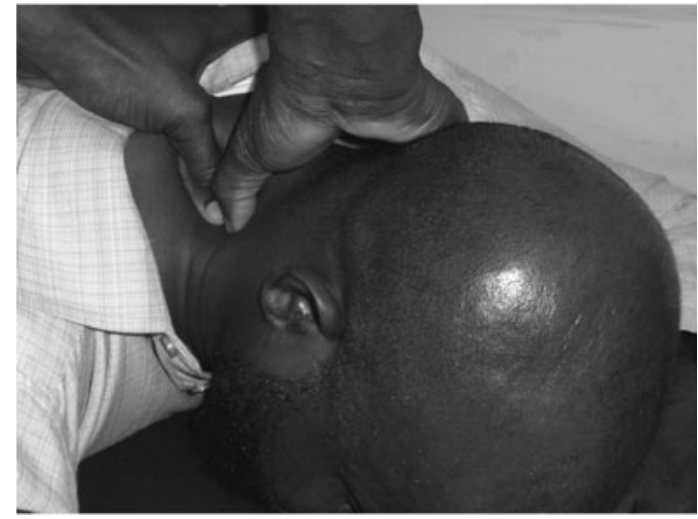

Fig. 1. Posterior-Anterior Unilateral Pressure (PAUP) to the $5^{\text {th }}$ cervical vertebra in a patient with right cervical spondylosis.

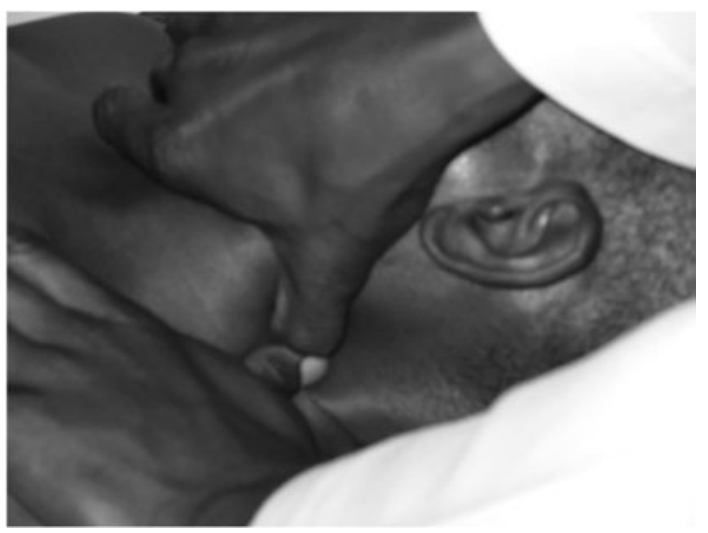

Fig. 2. Anterior-Posterior Unilateral Pressure (APUP) to $5^{\text {th }}$ cervical vertebra of a patient with left cervical spondylosis.

beyond the pain in an oscillatory manner every 6 seconds for 60 seconds (i.e. 10 oscillations) followed by a two-minute interval. This procedure was repeated on $\mathrm{C} 5$ and $\mathrm{C} 6$ respectively during each session for every patient in this group. Thus, the TT was $8 \mathrm{Y}(2 \mathrm{~min}$ treatment +2 min rest on $\mathrm{C} 5+2 \mathrm{~min}$ treatment +2 min rest on $\mathrm{C} 6+\mathrm{Y}$ ) where $\mathrm{Y}$ represent the variable time spent locating and initiating digital pressure and oscillation on C6.

Goup 2 (Fig. 2): Antero-Posterior Unilateral Pressure (APUP)

APUP was administered with the patient lying supine. Oscillatory pressure was directed through the thumbs of the therapist antero-posteriorly immediately over the transverse processes of C5 and $\mathrm{C} 6$ on the affected side. The digital pressure,

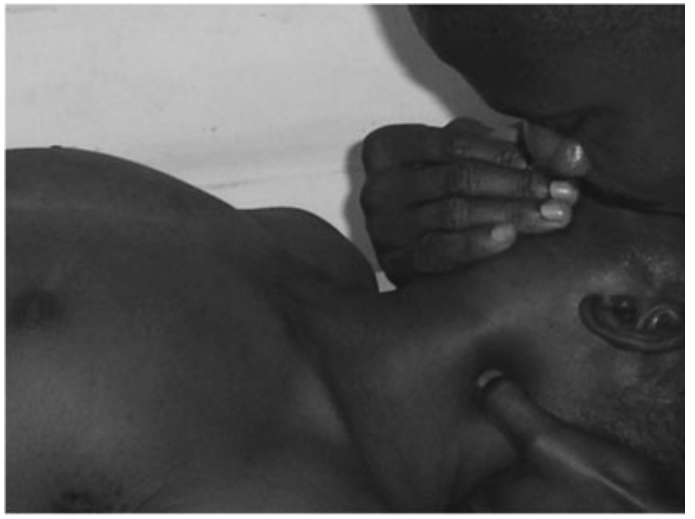

Fig. 3. Cervical Oscillatory Rotation (COR) to the right of a patient with left unilateral cervical spondylosis.

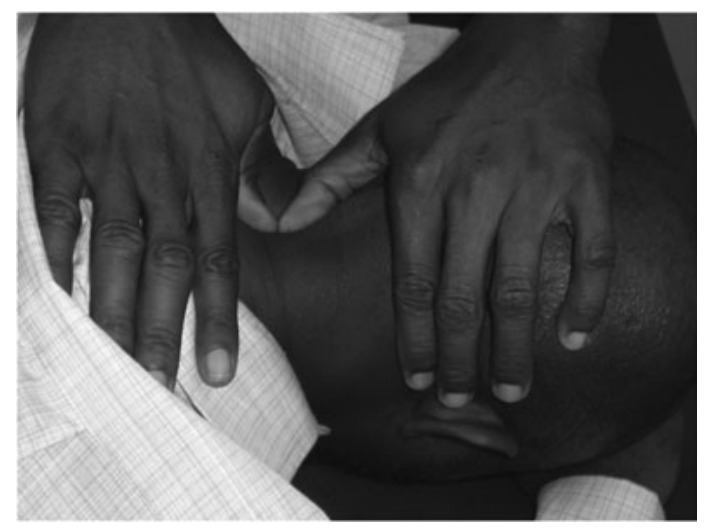

Fig. 4. Transverse Oscillatory Pressure (TOP) to the $5^{\text {th }}$ cervical vertebra of a patient with right unilateral cervical spondylosis.

oscillation pattern, frequency, latency and number of repetitions and TT were as for Group 1 above.

\section{Group 3 (Fig. 3): Cervical Oscillatory Rotation (COR)}

The patient also laid supine for COR. The therapist supported the patient's occiput which was jutting from the plinth with one hand, while grasping the jaw with the other hand. Treatment was effected by turning the head and neck to the side opposite the pain using both hands and then derotating in an oscillatory manner when the rotation just elicited pain or muscle spasm. The hand on the jaw determined the direction of rotation and derotation - right hand on the jaw when rotating to the patient's right and vice versa. The time between placement of the therapist's hands on the 
patient's occiput and the last oscillatory rotation was recorded as the TT. The oscillatory frequency, latency and duration were as for Group 1 above.

\section{Goup 4 (Fig. 4): Transverse Oscillatory Pressure (TOP)}

TOP was administered with the patient lying prone. The therapist's thumbs were placed on the side of the spinous process. Treatment was effected by a push-relax sequence on the spinous process using the thumbs to produce an oscillatory movement. Transverse pressure was directed towards the side of pain on C5 and C6 vertebra. The time between placement of the therapist's hands on the spinous process of $\mathrm{C} 5$ and the last oscillation on C6 was recorded as the TT. The oscillatory frequency, latency and duration were as for Group 1 above.

Stop watches (Eurastyle, Swiss made) were used to monitor the duration of each technique which was recorded after each session. The patients were on a thrice-weekly appointment schedule. All patients were treated by the same therapist who terminated their treatment when pain was no longer present. The maximum experimental treatment period for a patient was four weeks after which the total treatment time was estimated from the patient's record. Each patient's pain status was assessed by a trained neutral assessor, using a fivecategory scheme to determine if the patient was completely pain-free, had some residual pain, had fair improvement, pain was unchanged or was worse. This rating scheme was adopted because most patients in this environment commonly use these statements to describe their pain status. Besides, the visual analogue scale is not sufficiently sensitive to identify when an excruciating pain (10/ 10) gets worse ${ }^{13)}$. The data of patients (13 patients) who could not sustain treatment for four weeks while still in pain were excluded from this study. After three months, the patient's records were reviewed again to determine the number that needed to come back for treatment.

\section{DATA ANALYSIS}

The data of age and treatment time are reported as means \pm standard deviation (mean \pm S.D), while the number and percentage of patients in each of the sensory categories of the assessment scheme following treatment and relapse data are reported.
Analysis of variance was used to determine if significant differences existed in the categories of age, treatment time and number of patients in each pain state in the four treatment groups. If the F ratio was significant, a post hoc Scheffé procedure was used to determine which mean differences were significant. The SPSS statistical software (version 11) was utilized for data analysis on a TravelMate 4062NLCi Acer laptop computer. Significance was set at the 0.05 confidence level.

\section{RESULTS}

Table 1 displays the mean ages, mean treatment time, the number that returned for treatment after three months and pain recovery pattern of the four groups under consideration. No significant difference in age was found when the groups were compared with each other. However a significant difference was found between TT and number of pain-free patients in APUP $(\mathrm{TT}=130 \mathrm{~min}$, painfree $=63 \%)$ and PAUP $(\mathrm{TT}=161 \mathrm{~min}$, pain-free $=$ $46 \%$ ) groups. The TT and number of pain-free patients in the APUP and PAUP groups were significantly $(\mathrm{P}<0.001)$ different from those of the COR $(\mathrm{TT}=201 \mathrm{~min}$, pain-free $=17 \%)$ and TOP $(\mathrm{TT}=221 \mathrm{~min}$, pain-free $=25 \%)$ groups. These manual therapy techniques did not worsen the condition of patients but they had no effect on the symptoms of $4 \%$ and $8 \%$ of patients receiving TOP and COR, respectively. The relapse rates after three months in patients receiving TOP and COR respectively were $8 \%$ and $12 \%$.

\section{DISCUSSION}

From the empirical observations of Maitland ${ }^{7)}$ and $\mathrm{Nwuga}^{6}{ }^{6}$ the techniques used in this study were recommended for unilaterally distributed symptoms of cervical origin. The outcome measures in this study were pain recovery pattern, total TT needed for each technique, and rate of relapse within three months post treatment. The results show that not only was significantly less time spent in the use of pressure techniques (APUP and PAUP), but significantly more patients were pain-free with the use of these techniques when compared to COR and TOP. Going by these results one would be led to believe that for the signs and symptoms described, the choice of technique should be APUP and PAUP techniques. 
Table 1. Influence of mobilization techniques on treatment time, pain recovery and relapse pattern of patients with unilateral lower cervical spondylosis $(\mathrm{N}=96)$

\begin{tabular}{lcccc}
\hline & PAUP & APUP & COR & TOP \\
\hline Pain-free & $11^{\mathrm{a}}(46 \%)$ & $15^{\mathrm{b}}(63 \%)$ & $4^{\mathrm{c}}(17 \%)$ & $6^{\mathrm{c}}(25 \%)$ \\
Some Residual Pain & $8^{*}(33 \%)$ & $6^{*}(25 \%)$ & $9 *(29 \%)$ & $8^{*}(33 \%)$ \\
Fair Improvement & $5^{\mathrm{a}}(21 \%)$ & $3^{\mathrm{a}}(12 \%)$ & $8^{\mathrm{b}}(33 \%)$ & $9^{\mathrm{b}}(38 \%)$ \\
No Change & 0 & 0 & $2(8 \%)$ & $1(4 \%)$ \\
Worse & 0 & 0 & 0 & 0 \\
Total & $24(100 \%)$ & $24(100 \%)$ & $24(100 \%)$ & $24(100 \%)$ \\
Treatment Time (Minutes) & $161^{\mathrm{a}} \pm 9.3$ & $130^{\mathrm{b}} \pm 3.9$ & $201^{\mathrm{c}} \pm 9.2$ & $221^{\mathrm{c}} \pm 6.7$ \\
Number returning for treatment after 3 months & 0 & 0 & $3(12 \%)$ & $2(8 \%)$ \\
Age (Years) & $43.9^{*} \pm 2.2$ & $45.8^{*} \pm 3.23$ & $42.7^{*} \pm 2.9$ & $44.8^{*} \pm 3.0$ \\
\hline
\end{tabular}

PAUP, Posterior-Anterior Unilateral Pressure; APUP, Anterior-posterior Unilateral Pressure; COR, Cervical Oscillatory Rotation; TOP, Transverse Oscillatory Pressure.

In the pain-free and treatment time columns, numbers with superscript ' $a$ ' and ' $b$ ' are significantly $(\mathrm{P}<0.05)$ different, while numbers with superscript ' $c$ ' are not significantly $(\mathrm{P}>0.05)$ different, but are significantly $(\mathrm{P}<0.001)$ different from those with superscripts ' $a$ ' and ' $b$ '. Therefore, significantly less time was spent applying APUP technique than PAUP $(\mathrm{P}<0.05), \mathrm{COR}$ and TOP $(\mathrm{P}<0.001)$ to produce significantly more pain-free subjects at the same confidence limits. In the fair improvement column, numbers with superscript ' $a$ ' are not significantly $(\mathrm{P}>0.05)$ different and numbers with superscript ' $b$ ' are not significantly $(\mathrm{P}>0.05)$ different. However, numbers with superscript ' $b$ ' are significantly $(\mathrm{P}<0.05)$ more than those with 'a' superscript. *Not significantly $(\mathrm{P}>0.05)$ different.

Oscillatory pressure techniques of spinal mobilization therapy are known to have both neurophysiologic and mechanical effects ${ }^{11,12,14)}$. Paris ${ }^{14)}$ pointed out that mobilization technique stretches tissues by taking them into the area of plastic deformation of the stress-strain curve. The current findings indicate that oscillatory pressure techniques (APUP, PAUP, TOP) stretch cervical connective tissues and joint capsules to point $\mathrm{C}$ or $\mathrm{D}$ of the stress-strain curve, which is ideal for stretching tissues to produce a salvo of beneficial neuro inhibitory and mechanical effects ${ }^{11,12,14)}$. Nevertheless, the difficulty of stabilizing a transverse pressure on the spinous process (especially on obese patients) and the resistance offered by neck extensor muscle contraction to both TOP and PAUP seem to have constituted a hindrance that make it difficult to stretch the affected joint to point $\mathrm{C}$ (in some patients receiving TOP) and point $\mathrm{D}$ (in some patients receiving PAUP). Given the same intensity of pain therefore, APUP may facilitate the application of appropriate force magnitude during digital pressure and an adequate number of oscillations to mobilize the affected joints and stretch the soft tissues to achieve unrestricted and pain-free cervical motion, hence its superior analgesic potency over the other techniques featured in this study.

It was also observed that time spent on the APUP group was significantly $(\mathrm{P}<0.05)$ less than that spent on PAUP. Maitland ${ }^{7)}$ points out that care must be taken to ensure that the thumbs are positioned immediately over the transverse process for an effective unilateral pressure therapy. This study suggests that neck extensor muscular impedance is easier to overcome when pressure is applied antero-posteriorly, using the APUP technique than in PAUP, especially in the stocky, heavily built patient with a short thick neck that can be retracted into the thoracic area in a reflex effort of self protection by patients who are naïve to cervical manual therapy ${ }^{7,15)}$.

Oscillatory rotation (COR) may stretch the same tissues to point B of the stress strain curve of collagen ${ }^{14)}$. This stress is repeated by cyclic loading (i.e. a series of small stretches) producing only neurophysiological benefits, thus leaving patients with background joint play restrictions with a possibility of gradual build and flare-up of pain following activity. Added to this is the difficulty in getting the patient's neck at the appropriate angle to achieve the required facet apposition locking ${ }^{6}$, which may lead to insufficient oscillatory stress on the targeted joint and explain the low efficacy rating of the COR and TOP techniques.

Further studies comparing various other techniques for unilateral and bilaterally distributed symptoms should be endeavoured in the future. The 
information from this and future studies will assist physiotherapists and physiotherapy managers to adopt therapies which are effective for meeting the growing clinical challenges of cervical spondylosis.

\section{REFERENCES}

1) Galhom AA, Wagner FC: Cervical spondylosis. http:// www.emedicine.com/pmr/topic27.htm (Accessed Sep. 12, 2007).

2) Adams C: Out line of orthorpeadics. London: Churchillivingston, 2002.

3) McCarthy C: Priorities for research into the neurovascular complication of cervical spine manual therapy: Report of the MACP chair on a discussion session at the 2004 CSP AGM. Manual Therapy, 2006, 11: 85-86.

4) Creighton DS, Viti J, Krauss J: Use of translatoric mobilization in patient with cervical spondylotic degeneration: a case report. J Man Manip Ther, 2005, 13: 10-26.

5) Snodgrass SJ, Rivett DA, Robartson VJ: Manual forces applied during cervical mobilization. J Manipulative Physiol Ther, 2007, 30: 17-25.

6) Nwuga VC: Manipulation of the spine, Maryland:
Williams and Wilkins Company, 1976.

7) Maitland GD: Vertebral Manipulation, 5th edn. Boston: Butterworth-Hienemann 2003, pp201-212.

8) Zusman M: Spinal manipulative Therapy: Review of Some Proposed Mechanism, and a new hypothesis. Aust J Physiother, 1986, 32: 89-99.

9) DiFabio R: Efficacy of manual therapy. Phys Ther, 1991, 722: 853-864.

10) Mulligan BR: Manual Therapy. $5^{\text {th }}$ edition. Wellington: Plain view services ltd, 2004, pp9-15

11) Egwu MO, Alabi M, Nwuga VCB: Effect of Vertical Oscillatory pressure on neck pain and some cardiovascular variables. Physiotherapy, 2003, 89: 666-674

12) Egwu MO, Adewale AO, Olaogun MOB:The effect of vertical oscillatory pressure on youth and elderly adult low back pain intensity and lumbo-sacral mobility. JJPTA, 2007, 10: 17-26.

13) Charpman CR, Casey KL, Dubner R, et al.: Pain Measurement: an overview, Pain, 1985, 22: 1-31.

14) Paris SV: Mobilization of the spine. Phys Ther, 1979, 58: $988-995$.

15) Cook AJ, Brawer PA, Coalesce FM: The fearavoidance model of chronic pain: validation and age analysis using structural equation modeling. Pain, 2006, 121: 195-206. 